\title{
Change in Physical Activity and Stress During COVID-19 in a Sample of Older Adults with Exercise Habits
}

\author{
Petr Vajda ${ }^{1 *}$, Kateěina Strašilová ${ }^{2}$, Lenka Svobodová ${ }^{2}$ \\ ${ }^{1}$ Faculty of Arts, Masaryk university, Brno, Czech Republic; vajda@fsps.muni.cz \\ ${ }^{2}$ Faculty of Sports Studies, Masaryk University, Brno, Czech Republic; \\ strasilova@mail.muni.cz (K.S.), netyaloty@post.cz (L.S.)
}

\begin{abstract}
BACKGROUND: The COVID-19 outbreak forced many states to introduce public health and social measures (PHSM), which may pose an obstacle to performing physical activity (PA). METHODS: This study investigated PA levels and their changes and perceived stress in a sample of 193 participants (aged $71.21 \pm 4.87$ years) engaged in exercise lessons prior to PSHM. Data were collected via an online survey distributed directly to the participants. The survey included two instruments: the International Physical Activity Questionnaire and the Perceived Stress Scale.

RESULTS: The results indicate that sports habits may be a protective factor in staying active and meting $P A$ recommendations. Nevertheless, there were no associations between the number of exercise lessons in the pre-COVID-19 period and PA level or the perceived change in PA during PSHM. Ability to replace the cancelled exercise lesson with PA of similar duration and intensity was negatively associated with PA levels and its change. The PSS-10 score suggests that the sample did not experience a significant increase in perceived stress, but the relationship of this outcome to PA is unclear.

CONCLUSIONS: The promotion of alternative PA that could be performed during PHSM and building long-term exercise habits in older adults should be considered.
\end{abstract}

Keywords: SARS-CoV-2 Infection, Coronavirus Disease, Pandemic

\section{INTRODUCTION}

The worldwide COVID-19 pandemic forced many states to introduce public health and social measures (PHSM) to maintain social distance and reduce contact to protect people from exposure to infection of COVID-19. Measures such as lockdown, self-quarantine, restriction of outdoor movement, and closing of sport facilities could be challenges for physical activity (PA), perceived stress, or mental health (WHO 2020). The empirical research in older adults showed that COVID-19 PHSM are associated with negative changes in PA (Bourdas and Zacharakis 2020; Di Santo et al. 2020; Eek et al. 2021; Maugeri et al. 2020; Yamada et al. 2020a). This has also been corroborated in various samples of other age cohorts (e.g., (Barkley et al. 2020; Bourdas and Zacharakis 2020; Eek et al. 2021; Jia et al. 2020; Maugeri et al. 2020; Srivastav, Sharma, and Samuel 2021)). The worldwide pandemic has raised interest in the positive effects of PA on physical health. The reduction of PA resulting from established PHSM poses a significant dilemma, as PA is assumed to be a preventive factor against COVID-19 infection and other health issues. Recent reviews have concluded that PA and exercise may help promote health, improve immune response, and prevent chronic and viral infections in older adults. Therefore promoting PA may

\footnotetext{
* Corresponding author: Petr Vajda, Department of Psychology, Faculty of Arts, Masaryk university, Arna Nováka 1, Brno 602 00, Czech Republic; vajda@fsps.muni.cz; Tel.: +420 733603973 (CZ)
} 
be a good strategy in fighting COVID-19 (Amatriain-Fernández et al. 2020; Damiot et al. 2020; Hall and Church 2020; Sepúlveda-Loyola et al. 2020). Further, staying physically active during restriction is important for preventing future chronic conditions resulting from a sedentary lifestyle (Nyberg et al. 2020; Scartoni et al. 2020). Consistently, an empirical study of lifestyle risk factors showed that physical inactivity is a significant risk factor of severe infection (Hamer et al. 2020). The protective associations of PA on COVID-19 were observed even at relatively low levels of activity (i.e., < 150 min of moderate to vigorous activity) (Hamer et al. 2020).

Other significant aspects of staying physically active are mental health and well-being. The COVID-19 outbreak poses a threat to mental health linked to worries about self and close contact as well as to changes in life routines caused by PHSM. There is an increased risk of isolation for older people, as they are a vulnerable group in the case of COVID-19 illness. Studies investigating the impact of the COVID-19 pandemic and measures reveal that PA positively affects psychological health by enhancing self-esteem and resilience to stress, reducing depression and anxiety and having a positive impact on well-being (Carriedo et al. 2020; Lesser and Nienhuis 2020; Maugeri et al. 2020). Older adults engaged in vigorous and moderate-vigorous physical activity scored higher in self-efficacy, locus of control, and optimism and lower in depressive symptoms (Carriedo et al. 2020). Further, PA may positively affect cognitive function and consequently delay the progression of cognitive impairment in older adults. By contrast, depressive symptoms are associated with cognitive decline in older adults (Sachs-Ericsson et al. 2005). Therefore, staying physically active during the pandemic is important from both short- and long-term perspectives.

Roschel, Artioli, and Gualano (2020) considers the aggravation of PA as a relevant "adverse effect" of the PHSM taken to combat the spread of COVID-19. Despite the differences in PA and exercise recommendations, various global expert organisations have highlighted the importance of increasing and maintaining PA during the pandemic (Sepúlveda-Loyola et al. 2020). Therefore, governments and responsible experts should focus on the maintenance of PA levels, healthy lifestyles, and minimising the negative effects of PHSM on health and quality of life. Understanding the factors that may help older people stay active may be helpful in this issue. It is also important for potential future pandemics.

The current study investigated PA levels and their changes, the ability to replace the amount of time spent on exercise in currently closed facilities with another equally intense sports activity, and perceived stress in older people who were engaged in organised PA lessons prior to the COVID-19 outbreak. Thus, we determined whether exercise habits can help maintain the recommended level of PA during the pandemic.

We further hypothesise that: 1) there will be monotonic trends in PA levels and change in PA for an increasing number of days with more than 30 minutes of exercise prior to COVID-19 measures; and 2) there will be monotonic trends in PA levels and changes in PA for increasing replacement of organised PA lessons with similar PA.

\section{METHODS}

\section{Research Design and Participants}

This study targeted physically active people aged over 65 years who had been taking part in organised exercise lessons before COVID-19 outbreak. Data collection took place from 26 October 2020 to 9 November 2020 via an anonymous online questionnaire, which focused on exercises of older adults. The survey was distributed with the assistance of the staff of the facility used. Data sample consisted of clients from six different institutions. The questionnaire was sent directly to the participants' email addresses by the coaches or heads of facilities to avoid bias. Inclusion 
criteria were also checked by demographic and control questions (i.e., age and number of PA lessons per week in the pre-COVID-19 period). A total of 238 fully completed questionnaires were collected during the appointed period. Forty-five of them were excluded. Six respondents stated that the questionnaire was not intelligible for them, and 39 stated that they had serious health or mental problems that prevented them from physical activity. The final data sample consisted of 193 respondents (the description of the sample is provided in Table 1.

Table 1. Sample description

\begin{tabular}{|c|c|c|c|c|}
\hline${ }^{\star}$ PA status & $\begin{array}{c}\text { Low } \\
(\mathrm{n}=11)\end{array}$ & $\begin{array}{c}\text { Moderate } \\
(\mathrm{n}=83)\end{array}$ & $\begin{array}{c}\text { High } \\
(\mathrm{n}=99)\end{array}$ & $\begin{array}{c}\text { Total } \\
(\mathrm{n}=193)\end{array}$ \\
\hline Age (years) & $71.09 \pm 4.86$ & $71.45 \pm 5.36$ & $71.02 \pm 4.47$ & $71.21 \pm 4.87$ \\
\hline BMI & $26.37 \pm 4.41$ & $25.80 \pm 3.49$ & $25.62 \pm 4.04$ & $25.74 \pm 3.82$ \\
\hline Women $(\mathrm{N})$ & 8 & 72 & 89 & 169 \\
\hline $\operatorname{Men}(\mathrm{N})$ & 3 & 11 & 10 & 24 \\
\hline Living with a partner $(\mathrm{N})$ & 7 & 58 & 48 & 113 \\
\hline Living with partner separately $(\mathrm{N})$ & 3 & 5 & 12 & 20 \\
\hline Single $(N)$ & 1 & 20 & 39 & 60 \\
\hline Lives in a town with $>100000$ inhabitants $(\mathrm{N})$ & 10 & 72 & 81 & 163 \\
\hline Lives in a town $<100000$ inhabitants $(\mathrm{N})$ & & 4 & 4 & 8 \\
\hline Lives in the village $(\mathrm{N})$ & 1 & 7 & 14 & 22 \\
\hline Retire $(\mathrm{N})$ & 9 & 73 & 91 & 173 \\
\hline Working without restrictions due to COVID-19 (N) & 1 & 7 & 5 & 13 \\
\hline Working with restrictions due to COVID-19 (N) & 1 & 3 & 3 & 7 \\
\hline
\end{tabular}

* PA status was determined according to the International Physical Activity Questionnaire scoring.

\section{Government PHSM}

The study took place during the second wave of the pandemic. Government measures to prevent the spread of COVID-19 were adopted gradually. Indoor sports venues have been closed since 12 October 2020. Since October 22, the movement of people has been partially restricted, as well as the gathering of two or more people in public places or in groups up to 20 with a spacing of two meters. The operation of sports, cultural facilities, catering facilities, and most small shops was also limited. A curfew after 21:00 was introduced on October 26. Similar measures were adopted in the spring period during the first pandemic wave, so this was a second experience of lockdown during 2020.

\section{Data Collection}

The construction of the questionnaire followed a standard procedure (Passmore et al. 2002). The PA was assessed by a short version of the International Physical Activity Questionnaire (IPAQ), which allows the transfer of self-reported PA on METS-minutes. Considering the research sample age cohort, we followed recommendations for using the IPAQ in elderly people (Heesch et al. 2010). The self-reported change in physical activity between the previous week and the typical week of pre-COVID measures was assessed for total volume of PA and each category from the IPAQ (vigorous PA, moderate PA, walk, sitting time). The change was scored on a five-point scale ( $5=$ a lot more, $4=$ a bit more, $3=$ about the same, $2=$ a bit less, $1=a$ lot less). Considering 
the research sample, the scale was used in the form of a complete sentence, for example, "I was a lot more physically active". A similar scale was used to evaluate the perceived importance of PA during the pandemic in comparison to pre-pandemic period. Further, the questionnaire collected data about the usual frequency of physical exercise longer than 30 minutes prior to the COVID-19 measures and whether this exercise was compensated by another PA with a similar duration and intensity if it was provided by any closed organisation. Respondents stated the number of days with PA lessons, and we subsequently categorised it into four groups of days per week (1 day, 2-3 days, 4-5 days, 6-7 days. The replacement of organised PA was evaluated on a 4-point scale (not - 1; probably not - 2, probably yes -3 , yes -4 ), as well as perceived worry about COVID-19 infection. The 10-item Perceived Stress Scale (PSS-10) is a frequently used instrument to measure perceived stress. The PSS-10 measures the degree to which situations in one's life are appraised as stressful (Cohen, Kamarck, and Mermelstein 1983). We use the Czech version (Figalová 2019; Figalová and Charvát 2021) of the 10-item scale (PSS-10), which has been recommended for research usage by authors as adequate to the original 14-item version (Cohen 1988). The PSS-10 in this sample had acceptable to good internal consistency (Cronbach alpha 0.795). The questionnaire was supplemented by demographic and control questions.

\section{Data Analysis}

Statistical analysis was performed using IBM SPSS software (version 25). The normal distribution of the data was tested with the Shapiro-Wilk's test, $\alpha=.05$. A Jonckheere-Terpstra test $(\alpha=.05)$ was used to determine whether there were statistically significant increasing monotonic trends in examined variables. The medians are reported if the distributions of variables were similarly shaped for all groups, as assessed by visual inspection of a histogram. Results of variables with very dissimilar shapes of distributions are marked, as the Jonckheere-Terpstra test is not appropriate in such case (Laerd Statistics 2017). The Kendall's $\tau_{b}$ was used to determine the effect size for the Jonckheere-Terpstra test (Kraska-Miller 2019) and associations between variables. Average values are reported as mean + SD if the data had a normal distribution; otherwise, the mean with 95\% $\mathrm{CI}$ is reported. Descriptive data are presented in absolute and percentage frequencies.

\section{RESULTS}

Table 2 presents a summary of responses to questions related to physical activity and concern about COVID-19. The results showed that $89 \%$ of respondents did exercise or played sports two or more times per week and $40 \%$ even four or more times per week before COVID-19 measures. About $61 \%$ of respondents stated that they were probably not or were not able to replace the time spent on exercise in currently closed facilities with another equally intense sports activity. Despite this result, the research sample could be considered physically active even during COVID-19 measures, as only $6.7 \%$ of them were scored as low physically active by IPAQ. Table 2 shows that more than half of the participants (57\%) considered PA as more important during the COVID-19 pandemic, while only one respondent reported it as a bit less important. However, there was no significant association between the perceived importance of PA and its change (for all PA categories) or replacement of organised sport activities (Kendall's $\tau_{b} p>0.146$ ). 
Table 2. Distributions of responses related to PA and perceived worries about COVID-19 infection.

\begin{tabular}{lcccc}
\hline & 1 day & $2-3$ days & $4-5$ days & $6-7$ days \\
\hline $\begin{array}{l}\text { Number of days with exer- } \\
\text { cise longer than } 30 \text { min prior }\end{array}$ & $21(10.8 \%)$ & $93(47.7 \%)$ & $55(28.2 \%)$ & $24(12.3 \%)$ \\
$\begin{array}{l}\text { COVID-19 measures. }\end{array}$ & & &
\end{tabular}

\begin{tabular}{|c|c|c|c|c|c|}
\hline & not & \multicolumn{2}{|c|}{ probably not } & probably yes & yes \\
\hline $\begin{array}{l}\text { Replacement of time spend } \\
\text { on exercise in currently } \\
\text { closed facilities with another } \\
\text { equally intense sports } \\
\text { activity. }\end{array}$ & $30(15.4 \%)$ & $89(45.6 \%)$ & & $2.1 \%)$ & $31(15.9 \%)$ \\
\hline \multirow[t]{2}{*}{$\begin{array}{l}\text { Worries about COVID-19 } \\
\text { infection }\end{array}$} & $21(10.8 \%)$ & \multicolumn{2}{|c|}{$80(41.0 \%)$} & $58(29.7 \%)$ & $34(17.4 \%)$ \\
\hline & a lot less & a bit less & $\begin{array}{l}\text { about the } \\
\text { same }\end{array}$ & a bit more & a lot more \\
\hline Perception of importance of PA & 0 & $1(0.5 \%)$ & $80(41 \%)$ & $38(19.5 \%)$ & $74(37.9 \%)$ \\
\hline Change in total PA & $27(13.9 \%)$ & $46(23.6 \%)$ & $61(31.3 \%)$ & $34(17.4 \%)$ & $25(12.8 \%)$ \\
\hline Change in vigorous $\mathrm{PA}$ & $36(18.5 \%)$ & $38(19.5 \%)$ & $57(29.2 \%)$ & $35(17.9)$ & $27(13.8 \%)$ \\
\hline Change in moderate PA & $18(9.2 \%)$ & $39(20 \%)$ & $86(44.1 \%)$ & $33(16.9)$ & $17(8.7 \%)$ \\
\hline Change in walk & $25(12.8 \%)$ & $37(19 \%)$ & $71(36.4 \%)$ & $35(17.9)$ & $25(12.8 \%)$ \\
\hline Change in sitting time & $14(7.2 \%)$ & $19(9.7 \%)$ & $66(33.8 \%)$ & $55(28.2)$ & $39(20 \%)$ \\
\hline
\end{tabular}

The mean value of the PSS-10 score was 15.46, 95\% CI [14.73, 16.19]. Further analysis showed that there was a weak to moderate association between the PSS-10 score and perceived concerns about COVID-19 $\left(\tau_{\mathrm{b}}=.165, p=.002\right)$. On the contrary, no association between perceived concerns about COVID-19 and change in PA (for all categories) was found (Kendall's $\tau_{b} p>0.411$ ). Further, no association between PSS-10 score and PA levels or PA change was found (Kendall's $\left.\tau_{b} p>0.182\right)$.

\subsection{Hypothesised monotonic trends}

Our hypothesis that there would be monotonic trends in PA levels and change in PA for an increasing number of days with more than 30 minutes of exercise prior to COVID-19 PHSM was not supported (see Table 3). We also hypothesised that there would be monotonic trends in PA levels and changes in PA for increasing the replacement of organised PA lessons with PA of similar duration and intensity. A Jonckheere-Terpstra test determined that there was a statistically significant increasing monotonic trend in all variables related to PA or PA change in the four groups, dependent on the degree of replacement of prohibited PA lessons (see Table 3). However, there was no monotonic trend in sitting time, sitting time change, or PSS for either PA prior to COVID-19 or replacement organised PA lessons. 
Table 3. Hypothesised associations between pre-COVID-19 exercise, replacement of organised lessons and observed variables.

Number of days with exercise longer than 30 min pre-COVID 19 per week

\begin{tabular}{lcccccc}
\hline & 1 day & $2-3$ days & $4-5$ days & $6-7$ days & $\mathrm{p}$ & $\tau_{\mathrm{b}}$ \\
\hline Total PA $^{\#}$ & 3108 & 2826 & 3199.5 & 2139.5 & $0.315^{\mathrm{a}}$ & -0.027 \\
Total PA change $^{\#}$ & 3 & 3 & 3 & 3 & 0.204 & -0.05 \\
Vigorous PA $^{\#}$ & 640 & 720 & 960 & 800 & 0.224 & 0.043 \\
Vigorous PA change $^{\#}$ & 3 & 3 & 3 & 3 & 0.201 & -0.051 \\
Moderate PA $^{\#}$ & 600 & 600 & 720 & 680 & 0.489 & 0.002 \\
Moderate PA change $^{\#}$ & 3 & 3 & 3 & 3 & 0.343 & -0.025 \\
Walk $^{\#}$ & 1386 & 1039.5 & 1188 & 1039.5 & $0.151^{\mathrm{a}}$ & -0.058 \\
Walk change $^{\#}$ & 3 & 3 & 3 & 3 & 0.46 & -0.006 \\
Time sitting & 5 & 5 & 5 & 5 & 0.165 & 0.057 \\
Time sitting change & 4 & 3 & 4 & 4 & 0.213 & 0.049 \\
PSS & 16 & 14 & 16 & 15 & 0.437 & -0.009 \\
\hline
\end{tabular}

Replacement of organised lessons by another PA

\begin{tabular}{|c|c|c|c|c|c|c|}
\hline & 1 & 2 & 3 & 4 & $\mathrm{p}$ & $\tau_{b}$ \\
\hline Total $\mathrm{PA}^{\#}$ & 1677.00 & 2746.00 & 2826.00 & 4839.00 & $<0.001$ & $0.232^{* *}$ \\
\hline Total PA change $\mathrm{e}^{\#}$ & 2 & 2 & 3 & 3 & $<0.001$ & $0.203^{* *}$ \\
\hline Vigorous $\mathrm{PA}^{\#}$ & 400 & 640 & 960 & 1280.00 & 0.005 & $0.148^{\star *}$ \\
\hline Vigorous PA change ${ }^{\#}$ & & & & & $0.009^{b}$ & $0.142^{*}$ \\
\hline Moderate $\mathrm{PA}^{\#}$ & 510 & 540 & 640 & 960 & 0.015 & $0.121^{*}$ \\
\hline Moderate PA change ${ }^{\#}$ & 3 & 3 & 3 & 3 & 0.007 & $0.150^{*}$ \\
\hline Walk $^{\#}$ & 841.5 & 1039.50 & 1188.00 & 1584.00 & $<0.001^{\mathrm{a}}$ & $0.214^{* *}$ \\
\hline Walk change $e^{\#}$ & 3 & 3 & 3 & 3 & $0.01^{\mathrm{a}}$ & $0.141^{*}$ \\
\hline Time sitting ${ }^{\# \#}$ & 6 & 5 & 5 & 4 & $0.253^{\mathrm{a}}$ & -0.038 \\
\hline Time sitting change ${ }^{\# \#}$ & 4 & 4 & 4 & 3 & 0.385 & -0.107 \\
\hline PSS & 14 & 16 & 14 & 14 & 0.205 & -0.047 \\
\hline
\end{tabular}

${ }^{*}$ two-tailed $\mathrm{p}<0.05 ;{ }^{* *}$ two-tailed $\mathrm{p}<0.01{ }^{\text {a }}$ distributions are somewhat in shape, ${ }^{\mathrm{b}}$ distributions are very dissimilar in shape, data are presented as median.

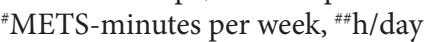

\section{DISCUSSION}

There is opportunity in every crisis, and the worldwide COVID-19 pandemic is not an exception. Therefore, researchers should focus on both the potential risk and protective factors to help manage not only the current situation, but also those of the future. The sample of participants over 65 years of age who were engaged in regular organised PA lessons prior to the COVID-19 outbreak was assessed in the current study. This allowed us to examine the extent to which an exercise habit could be a protective factor in staying physically active during pandemics or social isolations. The results showed that only $5.7 \%$ of the included participants reported low PA according to IPAQ scoring (Anon 2005). Moreover, more than half of the sample (51.3\%) reported high PA. As the scoring of the moderate PA category by IPAQ was similar to WHO recommendations for PA in older adults, it could be assumed that most of the sample met WHO PA requirements (WHO 2020). WHO recommends a minimum level of PA to improve cardiorespiratory and muscular fitness, bone and functional health, and reduce the risk of NCDs, depression, and 
cognitive decline. For additional health benefits, adults should increase their PA above the minimum requirement (Organisation mondiale de la santé 2010). Therefore, regardless of whether the participants experienced a decrease or increase in PA after the COVID-19 outbreak, it can be concluded that most of them met the minimum requirements for PA to maintain health during the PHSM. The sample in this study (older than 65 years) reported a high average level of PA (3554 $\pm 2858 \mathrm{MET}-\mathrm{min} /$ week; mean). As a comparison, a study of the Italian population using the IPAQ reported a mean of $1473 \mathrm{MET}$-min/week in people over 60 years (Maugeri et al. 2020), Portuguese older adults > 65 years, $2448 \pm 3435$ MET-min/week (Antunes et al. 2020), and $47.6 \%$ Italian older adults (aged $>60$ years) with mild cognitive impairment or subjective cognitive decline were in the low physical activity category (<600 MET-min/week) (Di Santo et al. 2020). Another study that used IPAQ to examine the change in PA after the COVID-19 outbreak in Japanese community-dwelling older adults reported a median (IQR) of total PA time in minutes per week of 330 (148-600) for "robust" and 270 (120-480) for "pre-frail" subgroups" (Yamada et al. 2020a), which is less than half of that of the sample in this study $684(420-1080)$. The research tools used do not allow the assessment of physical fitness, but the mean BMI of the sample (25.74 \pm 3.82$)$ was in the optimal range (23.0 to 29.9) associated with optimal longevity (Porter Starr and Bales 2015). This result suggests that older adults with the habit of exercising and engaging in organised lessons may have a greater tendency to stay physically active than the general older population when obstacles appear. However, it must be taken into consideration that the results come from a population of different socio-cultural backgrounds, and the data were collected under different PHSM.

Aside from adherence to recommendations, there is a question of changes in PA patterns during the PSHM. Prior research reported a decrease in PA levels (Bourdas and Zacharakis 2020; Di Santo et al. 2020; Eek et al. 2021; Maugeri et al. 2020; Yamada et al. 2020a) in older adults, except a study on British community dwelling individuals (Richardson et al. 2020). In this study, respondents reported an increase in total PA and each subcategory in approximately $22.9-32.1 \%$ cases, whereas a decrease was observed in $29.5-38.3 \%$ cases (see Table 2). We hypothesised that there would be monotonic trends in PA levels and change in PA for an increasing number of days with more than 30 minutes of exercise prior to COVID-19 measures (i.e., level of habitual exercise). Contrary to expectations, this study did not find such a trend in any of the PA categories, or even in sitting time (see Table 3). Therefore, it is possible that habitual exercise or sports activity is a protective factor for staying physically active when obstacles occur; however, there was no monotonic trend between frequency of exercise and levels or changes in PA during pandemic. This result is limited by the absence of investigation of the impact of PHSM on various aspects of the participants' lives, which can play an important role in PA levels. Further, it can be assumed that the exercise represented a different portion of the total PA for each individual.

We assume that the extent to which seniors are able to cope with pandemic measures plays an important role in their PA. All participants in this study engaged in organised PA lessons, which were cancelled due to social isolation. We hypothesised that the ability to replace exercise lessons with PA of similar intensity and duration is an important factor in PA patterns during the period of PHSM. Our results revealed a monotonic trend in both the level of PA and its change after introduction PHSM depending on the ability to replace the organised exercise (see Table 3 ). Effect size was determined by $\tau_{b}$ and ranged from 0.141 to 0.232 for different categories of PA, which represented weak to moderate association. This means that those who were less able to replace their usually organised exercise were less active and more often perceived a decrease in their physical activity in comparison to the pre-COVID-19 period. Moreover, $48.7 \%$ of participants reported an increase in sitting time, while only $17 \%$ declared spending less time sitting than in the pre-COVID-19 period (see Table 3). No association was found between change in sitting 
time and replacement of organised exercise or number of days in which participant exercised in the pre-COVID-19 period. Increasing the time spent sitting while maintaining the level of PA was also reported by Richardson at al. (2020) in a sample of community-dwelling individuals aged $>70$ years. This result suggests that although the exercise habit may be a protective factor in maintaining PA, the promotion of suitable exercise during PHSM is required, as well as a focus on preventing sedentary behaviour. This may be rather challenging, as the great emphasis in PA promotion programmes is placed on digital technology, which is longstanding less used by older adults (see "age-based digital divide" (Martins Van Jaarsveld 2020)). Nevertheless, governments should prepare strategies to minimise the impact of closing sports facilities.

We consider the study by Richardson et al. (2020) interesting because, unlike most of the results, it highlights maintaining a level of PA in a sample of older adults. Authors had offered an interesting explanatory factors which may played role. The participants in the sample perceived the high importance of staying physically active. Average marks ranged from 8.8 to 9.1 at different time points of the study, where the rating scale was 1-10 (10 being extremely important). Further, the authors pointed to some situational factors, such as good weather conditions and an increase in time spent in gardens, as gardening was the second most popular change to activities. Our finding is consistent with the mentioned study. As our sample population was engaged in organised PA lessons prior to the COVID-19 outbreak, it can be assumed that they found PA as important. Moreover, 58\% of the participants stated that they perceived PA as more important since the COVID-19 outbreak, and only one participant perceived PA as slightly less important. Therefore, it is possible that the perceived importance of PA could affect the high level of PA that the research sample reached. Nevertheless, there was no association between changes in the perceived importance of PA and its level or change. Regarding the situational factors, while the weather and sufficient gardening conditions possibly positively affected the PA levels in the study mentioned above (Richardson et al. 2020), our results show a negative association between the perceived replacement of organised PA lessons and PA levels and its change in all categories. The role of situational conditions is also supported by the study of a Japanese sample of older adults (age $73.9 \pm 5.6$ years), which shows that after lifting the state of emergency and subsequently partially bringing conditions back to the pre-COVID-19 state, the level of PA recovered to the pre-COVID-19 state in most of the participants (except those who were living alone and socially inactive) (Yamada et al. 2020b). In this light, future research and governments should focus on the perceived importance of PA by older adults and on maintaining appropriate conditions for the implementation of PA during PHSM.

The COVID-19 pandemic and the connected PHSM can be considered a stressful period. Hence, we investigated perceived stress to determine whether PA habit can be a protective factor. There are no conclusively adapted cut-off scores for PSS-10 (the authors of the scale did not establish any), and it has been suggested that it should be used in comparison with different samples (Remor 2006). Generally, PSS-10 score tends to lower with age (Cohen and JanickiDeverts 2012), and despite being at higher risk, older people report lower stress (Adamson et al. 2020). This tendency also occurred in the Czech standardisation study, which showed a negative correlation between age and PSS-10 score $\mathrm{r}=-.34, \mathrm{p}<0.001$. The mean PSS-10 score in the Czech population sample was $18.04 \pm 6.83$ (mean \pm SD) (Figalová 2019), which is higher than that of this study (mean $=15.46,95 \%$ CI $[14.73,16.19])$. It should be considered that women in the Czech population perceived greater stress $(19.12 \pm 6.75)$ than men $(16.62 \pm 6.68)$, and the sample in this study consisted of $87.6 \%$ women (Figalová 2019). Similar trend was in current sample $($ men $=13.83 \pm 4.83$, women $=15.69 \pm 5.14)$. Nevertheless, this result is limited by small proportion of men in the sample $(n=24)$. 
Interpretation of this result is problematic as we do not have data from the period before COVID-19 outbreak. An interesting comparison may be provided by the comprehensive dataset from the COVIDiSTRESS global survey. This study included a Czech sample of 1995 participants who reported $2.694 \pm 0.707$ (mean \pm SD) (COVIDiSTRESS Global Survey Consortium et al. 2021), which is much higher than the Czech mean value before COVID-19. The mentioned value is the mean for a single PSS item; therefore, for comparison, it is necessary to multiply it by ten. This indicates that there could be an increase in perceived stress linked to the COVID-19 outbreak in the Czech population, which may be due to the strict long-term PHSM introduced by government. Based on our results, we assumed that no similar change was detected in the current sample. It is important to bear in mind that there are significant limitations to this assumption. First, the COVIDiSTRESS sample was younger (33.4 \pm 11.5 years) than the population in the Czech PSS-10 standardisation study (44.3 \pm 12.8 years) (Figalová 2019) and much younger than the current sample. Further, the COVIDiSTRESS global survey investigated different pandemic periods between 30 March and 30 May 2020. The current study excluded respondents who were unable to do PA due to health issue. Moreover, no association was found between perceived stress and PA level or reported change in PA, number of days with exercise longer than 30 min pre-COVID 19 per week, or replacement of organised lessons with another PA. Therefore, it is not suitable to make any conclusions about the relation between PA and perceived stress in this sample.

Aside from already reported limitations, it is necessary to mention that the results are based on self-reported perceptions of change in PA, which is not standardised, and IPAQ is an indirect measurement tool. To minimise the misunderstanding of the questions and their meaning, we ran a pilot study. Further, we did not assess the individual impact of the COVID-19 pandemic and PHSM, but it can be assumed that each participant was touched by the situation differently (e.g., different obstacles in usual daily routine, health issues of close person, systemic stress load in family).

\section{CONCLUSIONS}

The current study demonstrated that sport habits can help to maintain a sufficient level of sports activity necessary to promote health in people aged over 65 when pandemic PHSM are introduced (sport venues closed, restricted movement and gathering two or more people in public place etc.). Nevertheless, there was no association between the number of days with more than 30 minutes of exercise activity in the pre-COVID-19 period and reported PA or perceived PA changes during the period of PHSM. The negative association between the reported ability to replace the cancelled organised exercise lessons and PA and its perceived change during PHSM highlights the importance of providing opportunities and ways to replace the PA that cannot be carried out. Despite the limitations of the study design and the impossibility of comparing data with another study on the Czech population of the same age cohort, the results suggest that the participants in the current sample did not experience a significant increase in perceived stress. However, this may be related to age, as there was no association between PA variables and PSS-10 scores.

Funding: This research did not receive any specific grants from funding agencies in the public, commercial, or not-for-profit sectors.

Data Availability Statement: Data are available on request (vajda@fsps.muni.cz).

Conflicts of Interest: The authors declare no conflict of interest. 
Ethics: The study conformed to the Declaration of Helsinki. Ethical review and approval were waived for this study, due to research was fully anonymous and voluntary.

\section{References}

Adamson, Maheen M., Angela Phillips, Srija Seenivasan, Julian Martinez, Harlene Grewal, Xiaojian Kang, John Coetzee, Ines Luttenbacher, Ashley Jester, Odette A. Harris, and David Spiegel. 2020. 'International Prevalence and Correlates of Psychological Stress during the Global COVID-19 Pandemic'. International Journal of Environmental Research and Public Health 17(24):9248. doi: 10.3390/ijerph17249248.

Amatriain-Fernández, Sandra, Thomas Gronwald, Eric Murillo-Rodríguez, Claudio Imperatori, Alexandre Francisco Solano, Alexandra Latini, and Henning Budde. 2020. 'Physical Exercise Potentials Against Viral Diseases Like COVID-19 in the Elderly'. Frontiers in Medicine 7:379. doi: 10.3389/fmed.2020.00379.

Anon. 2005. 'Guidelines for Data Processing and Analysis of the International Physical Activity Questionnaire (IPAQ) - Short and Long Forms'.

Antunes, Raul, Roberta Frontini, Nuno Amaro, Rogério Salvador, Rui Matos, Pedro Morouço, and Ricardo Rebelo-Gonçalves. 2020. 'Exploring Lifestyle Habits, Physical Activity, Anxiety and Basic Psychological Needs in a Sample of Portuguese Adults during COVID-19'. International Journal of Environmental Research and Public Health 17(12):4360. doi: 10.3390/ ijerph17124360.

Barkley, Jacob E., Andrew Lepp, Ellen Glickman, Greg Farnell, Jake Beiting, Ryan Wiet, and Bryan Dowdell. 2020.'The Acute Effects of the COVID-19 Pandemic on Physical Activity and Sedentary Behavior in University Students and Employees'. International Journal of Exercise Science 13(5):1326-39.

Bourdas, Dimitrios I., and Emmanouil D. Zacharakis. 2020. 'Impact of COVID-19 Lockdown on Physical Activity in a Sample of Greek Adults'. Sports 8(10):139. doi: 10.3390/sports8100139.

Carriedo, Alejandro, José A. Cecchini, Javier Fernandez-Rio, and Antonio Méndez-Giménez. 2020. 'COVID-19, Psychological Well-Being and Physical Activity Levels in Older Adults During the Nationwide Lockdown in Spain'. The American Journal of Geriatric Psychiatry: Official Journal of the American Association for Geriatric Psychiatry 28(11):1146-55. doi: 10.1016/j.jagp.2020.08.007.

Cohen, Sheldon. 1988. 'Perceived Stress in a Probability Sample of the United States.' Pp. 31-67 in The social psychology of health., The Claremont Symposium on Applied Social Psychology. Thousand Oaks, CA, US: Sage Publications, Inc.

Cohen, Sheldon, and Denise Janicki-Deverts. 2012. 'Who's Stressed? Distributions of Psychological Stress in the United States in Probability Samples from 1983, 2006, and 20091: PSYCHOLOGICAL STRESS IN THE U.S.' Journal of Applied Social Psychology 42(6):1320-34. doi: 10.1111/j.1559-1816.2012.00900.x.

Cohen, Sheldon, Tom Kamarck, and Robin Mermelstein. 1983. 'A Global Measure of Perceived Stress'. Journal of Health and Social Behavior 24(4):385. doi: 10.2307/2136404.

COVIDiSTRESS Global Survey Consortium, Yuki Yamada, Dominik-Borna Ćepulić, Tao Coll-Martín, Stéphane Debove, Guillaume Gautreau, Hyemin Han, Jesper Rasmussen, Thao P. Tran, Giovanni A. Travaglino, and Andreas Lieberoth. 2021. 'COVIDiSTRESS Global Survey Dataset on Psychological and Behavioural Consequences of the COVID-19 Outbreak'. Scientific Data 8(1):3. doi: 10.1038/s41597-020-00784-9.

Damiot, Anthony, Ana Jéssica Pinto, James E. Turner, and Bruno Gualano. 2020. 'Immunological Implications of Physical Inactivity among Older Adults during the COVID-19 Pandemic'. Gerontology 66(5):431-38. doi: 10.1159/000509216.

Di Santo, Simona Gabriella, Flaminia Franchini, Beatrice Filiputti, Angela Martone, and Serena Sannino. 2020. 'The Effects of COVID-19 and Quarantine Measures on the Lifestyles and Mental Health of People Over 60 at Increased Risk of Dementia'. Frontiers in Psychiatry 11:578628. doi: 10.3389/fpsyt.2020.578628.

Eek, Frida, Caroline Larsson, Anita Wisén, and Eva Ekvall Hansson. 2021. 'Self-Perceived Changes in Physical Activity and the Relation to Life Satisfaction and Rated Physical Capacity in Swedish Adults during the COVID-19 Pandemic-A Cross Sectional Study'. International Journal of Environmental Research and Public Health 18(2):671. doi: 10.3390/ijerph18020671.

Figalová, Nikol. 2019. 'PŘEKLAD A PSYCHOMETRICKÉ VLASTNOSTI ČESKÉ VERZE ŠKÁLY VNÍMANÉHO STRESU'. master thesis, Univerzita Palackého v Olomouci, Filozofická fakulta, Olomouc.

Figalová, Nikol, and Miroslav Charvát. 2021. 'The Perceived Stress Scale: Reliability and Validity Study in the Czech Republic'. Ceskoslovenska Psychologie 65(1):46-59. doi: 10.51561/cspsych.65.1.46.

Hall, Mary-Frances E., and Frank C. Church. 2020. 'Exercise for Older Adults Improves the Quality of Life in Parkinson's Disease and Potentially Enhances the Immune Response to COVID-19'. Brain Sciences 10(9):612. doi: 10.3390/brainsci10090612.

Hamer, Mark, Mika Kivimäki, Catharine R. Gale, and G. David Batty. 2020. 'Lifestyle Risk Factors, Inflammatory Mechanisms, and COVID-19 Hospitalization: A Community-Based Cohort Study of 387,109 Adults in UK'. Brain, Behavior, and Immunity 87:184-87. doi: 10.1016/j.bbi.2020.05.059.

Heesch, Kristiann C., Jannique GZ van Uffelen, Robert L. Hill, and Wendy J. Brown. 2010. 'What Do IPAQ Questions Mean to Older Adults? Lessons from Cognitive Interviews'. International Journal of Behavioral Nutrition and Physical Activity 7(1):35. doi: 10.1186/1479-5868-7-35. 
STUDIA SPORTIVA 2021/2

Jia, Peng, Lei Zhang, Wanqi Yu, Bin Yu, Meijing Liu, Dong Zhang, and Shujuan Yang. 2020. 'Impact of COVID-19 Lockdown on Activity Patterns and Weight Status among Youths in China: The COVID-19 Impact on Lifestyle Change Survey (COINLICS)'. International Journal of Obesity. doi: 10.1038/s41366-020-00710-4.

Kraska-Miller, M. 2019. Nonparametric Statistics for Social and Behavioral Sciences. Place of publication not identified: CRC Press.

Laerd Statistics. 2017. 'Jonckheere-Terpstra Test Using SPSS Statistics.' Statistical Tutorials and Software Guides. Retrieved (https://statistics.laerd.com/).

Lesser, Iris A., and Carl P. Nienhuis. 2020. 'The Impact of COVID-19 on Physical Activity Behavior and Well-Being of Canadians'. International Journal of Environmental Research and Public Health 17(11):3899. doi: 10.3390/ijerph17113899.

Martins Van Jaarsveld, Gabrielle. 2020. 'The Effects of COVID-19 Among the Elderly Population: A Case for Closing the Digital Divide.' Frontiers in Psychiatry 11:577427. doi: 10.3389/fpsyt.2020.577427.

Maugeri, Grazia, Paola Castrogiovanni, Giuseppe Battaglia, Roberto Pippi, Velia D’Agata, Antonio Palma, Michelino Di Rosa, and Giuseppe Musumeci. 2020. 'The Impact of Physical Activity on Psychological Health during Covid-19 Pandemic in Italy'. Heliyon 6(6):e04315. doi: 10.1016/j.heliyon.2020.e04315.

Nyberg, Solja T., Archana Singh-Manoux, Jaana Pentti, Ida E. H. Madsen, Severine Sabia, Lars Alfredsson, Jakob B. Bjorner, Marianne Borritz, Hermann Burr, Marcel Goldberg, Katriina Heikkilä, Markus Jokela, Anders Knutsson, Tea Lallukka, Joni V. Lindbohm, Martin L. Nielsen, Maria Nordin, Tuula Oksanen, Jan H. Pejtersen, Ossi Rahkonen, Reiner Rugulies, Martin J. Shipley, Pyry N. Sipilä, Sari Stenholm, Sakari Suominen, Jussi Vahtera, Marianna Virtanen, Hugo Westerlund, Marie Zins, Mark Hamer, G. David Batty, and Mika Kivimäki. 2020. 'Association of Healthy Lifestyle With Years Lived Without Major Chronic Diseases'. JAMA Internal Medicine 180(5):760. doi: 10.1001/jamainternmed.2020.0618.

Organisation mondiale de la santé. 2010. Global Recommendations on Physical Activity for Health. Genève: WHO.

Passmore, Cindy, Alison E. Dobbie, Michael Parchman, and James Tysinger. 2002. 'Guidelines for Constructing a Survey'. Family Medicine 34(4):281-86.

Porter Starr, Kathryn N., and Connie W. Bales. 2015. 'Excessive Body Weight in Older Adults'. Clinics in Geriatric Medicine 31(3):311-26. doi: 10.1016/j.cger.2015.04.001.

Remor, Eduardo. 2006. 'Psychometric Properties of a European Spanish Version of the Perceived Stress Scale (PSS)'. The Spanish Journal of Psychology 9(1):86-93. doi: 10.1017/S1138741600006004.

Richardson, Darren L., Michael J. Duncan, Neil D. Clarke, Tony D. Myers, and Jason Tallis. 2020. 'The Influence of COVID-19 Measures in the United Kingdom on Physical Activity Levels, Perceived Physical Function and Mood in Older Adults: A Survey-Based Observational Study'. Journal of Sports Sciences 1-13. doi: 10.1080/02640414.2020.1850984.

Roschel, Hamilton, Guilherme G. Artioli, and Bruno Gualano. 2020. 'Risk of Increased Physical Inactivity During COVID -19 Outbreak in Older People: A Call for Actions'. Journal of the American Geriatrics Society 68(6):1126-28. doi: 10.1111/ jgs. 16550 .

Sachs-Ericsson, Natalie, Thomas Joiner, E. Ashby Plant, and Dan G. Blazer. 2005. 'The Influence of Depression on Cognitive Decline in Community-Dwelling Elderly Persons'. The American Journal of Geriatric Psychiatry 13(5):402-8. doi: 10.1097/00019442-200505000-00009.

Scartoni, Fabiana Rodrigues, Leandro de Oliveira Sant'Ana, Eric Murillo-Rodriguez, Tetsuya Yamamoto, Claudio Imperatori, Henning Budde, Jeferson Macedo Vianna, and Sergio Machado. 2020. 'Physical Exercise and Immune System in the Elderly: Implications and Importance in COVID-19 Pandemic Period'. Frontiers in Psychology 11:593903. doi: 10.3389/ fpsyg.2020.593903.

Sepúlveda-Loyola, W., I. Rodríguez-Sánchez, P. Pérez-Rodríguez, F. Ganz, R. Torralba, D. V. Oliveira, and Leocadio RodríguezMañas. 2020. 'Impact of Social Isolation Due to COVID-19 on Health in Older People: Mental and Physical Effects and Recommendations.' The Journal of Nutrition, Health \& Aging. doi: 10.1007/s12603-020-1469-2.

Srivastav, Adarsh Kumar, Neha Sharma, and Asir John Samuel. 2021. 'Impact of Coronavirus Disease-19 (COVID-19) Lockdown on Physical Activity and Energy Expenditure among Physiotherapy Professionals and Students Using Web-Based Open E-Survey Sent through WhatsApp, Facebook and Instagram Messengers'. Clinical Epidemiology and Global Health 9:78-84. doi: 10.1016/j.cegh.2020.07.003.

WHO. 2020. 'Stay Physically Active during Self-Quarantine'. World Health Organization Regional Office for Europe. Retrieved 29 December 2020 (https://www.euro.who.int/en/health-topics/health-emergencies/coronavirus-covid-19/publicationsand-technical-guidance/noncommunicable-diseases/stay-physically-active-during-self-quarantine).

Yamada, Minoru, Y. Kimura, D. Ishiyama, Y. Otobe, M. Suzuki, S. Koyama, T. Kikuchi, H. Kusumi, and H. Arai. 2020a. 'Effect of the COVID-19 Epidemic on Physical Activity in Community-Dwelling Older Adults in Japan: A Cross-Sectional Online Survey'. The Journal of Nutrition, Health \& Aging. doi: 10.1007/s12603-020-1424-2.

Yamada, Minoru, Y. Kimura, D. Ishiyama, Y. Otobe, M. Suzuki, S. Koyama, T. Kikuchi, H. Kusumi, and H. Arai. 2020b.'Recovery of Physical Activity Among Older Japanese Adults Since the First Wave of the COVID-19 Pandemic'. The Journal of Nutrition, Health \& Aging. doi: 10.1007/s12603-020-1466-5. 